\title{
Primary non-syndromic lymphoedema (Meige disease) is not caused by mutations in FOXC2
}

\author{
Tayebeh Rezaie $^{1}$, Rose Ghoroghchian ${ }^{1}$, Rachel Bell ${ }^{2}$, Glen Brice ${ }^{2}$, Ali Hasan ${ }^{3}$, \\ Kevin Burnand ${ }^{4}$, Steve Vernon ${ }^{5}$, Sahar Mansour ${ }^{2}$, Peter Mortimer ${ }^{3}$, Steve Jeffery ${ }^{2}$, \\ Anne Child ${ }^{3}$ and Mansoor Sarfarazi ${ }^{*, 1}$
}

\begin{abstract}
${ }^{1}$ Molecular Ophthalmic Genetics Laboratory, Department of Surgery, University of Connecticut Health Center, Farmington, CT, USA; ${ }^{2}$ Department of Medical Genetics, St George's University of London, London, UK; ${ }^{3}$ Department of Cardiac and Vascular Sciences, St George's University of London, London, UK; ${ }^{4}$ Department of Vascular Surgery, Guy's and St Thomas' NHS Hospital Trust, London, UK; ${ }^{5}$ Department of Ophthalmology, University Hospital, Queen's Medical Center, Nottingham, UK
\end{abstract}

Primary lymphoedema is a genetic disorder with numerous phenotypic subgroups. The most common form is the non-syndromic Meige disease, which is primarily of pubertal or later onset, with oedema clinically indistinguishable from that found in the lymphoedema-distichiasis syndrome. There are also other very rare forms of lymphoedema such as yellow nail syndrome and lymphoedema with ptosis, which are clinically similar to Meige disease. The only causative genes so far identified for the non-congenital primary lymphoedemas are the transcription factor $F O X C 2$, where mutations are known to produce lymphoedema with distichiasis, and SOX18 in the very rare condition hypotrichosis-lymphoedematelangiectasia. This study has examined FOXC2 gene by sequence analysis in 23 affected individuals with Meige disease. A novel truncating mutation (c.563-584del) was identified in one family and found to segregate with the disease in eight affected relatives over three generations. This deletion creates a frameshift that predicts a premature stop at nucleotide 599 and truncating the normal protein by $38 \%$. Although the affected patient initially selected for mutation screening from this family had lymphoedema without distichiasis, all but one of his affected relatives who carried the FOXC2 mutation did have accessory eyelashes originating from their meibomian glands. This is further confirmation that of the primary lymphoedemas, only lymphoedema with distichiasis is caused by FOXC2 mutations. All forms of postpubertal lymphoedema need careful phenotyping for distichiasis, which may prove difficult to confirm unless several family members are examined, and cannot ever be assumed to be absent from self-report. European Journal of Human Genetics (2008) 16, 300-304; doi:10.1038/sj.ejhg.5201982; published online 16 January 2008

Keywords: distichiasis; FOXC2; lymphoedema

\footnotetext{
*Correspondence: Professor M Sarfarazi, Molecular Ophthalmic Genetics Laboratory, Department of Surgery, University of Connecticut Health Center, 263 Farmington Avenue, Farmington, CT 06032-1110, USA. Tel: + 1860679 3629; Fax: + 18606797524 ;

E-mail: Mansoor@Neuron.uchc.edu

Received 24 July 2007; revised 23 October 2007; accepted 24 October 2007; published online 16 January 2008
}

Introduction

Lymphoedema is a chronic tissue swelling, most frequently of the lower limbs, resulting from deficient lymphatic drainage in the presence of normal capillary function. ${ }^{1}$ Primary lymphoedema is caused by an intrinsic abnormality of the lymph conducting pathways, and can be present from birth (Milroy disease (MD), OMIM 153100), or can develop later, often at or after puberty (Meige disease (MGD), OMIM 153200, and lymphoedema distichiasis 
(LD), OMIM 153400). Although LD and MGD share clinically indistinguishable lymphoedema, MGD is nonsyndromic whereas LD presents with a range of symptoms. These include secondary eyelashes originating from the meibomian glands in $94 \%$ of those with mutations, early varicose veins (49\%), ptosis (31\%), cleft palate $(4 \%)$ and cardiac abnormalities. ${ }^{1}$ There is also venous reflux in $100 \%$ of those individuals with a FOXC2 mutation, regardless of their lymphoedema status. ${ }^{2}$

MD has been mapped to $5 \mathrm{q} 35^{3}$ and the gene identified as VEGFR $3,{ }^{4}$ and a locus for LD was discovered on $16 \mathrm{q} 24.3,{ }^{5}$ where mutations in FOXC2 were found to cause the disorder. $^{6-8}$ There is as yet no gene or locus identified for MGD. Two very rare forms of primary lymphoedema have been proposed to exist; lymphoedema associated with discoloured, slow growing and excessively curved nails (lymphoedema and yellow nail syndrome (YNS); OMIM 153300), and lymphoedema with ptosis (OMIM 153000). Whether these truly exist as separate entities is open to question, as yellow nails can be found in MD, MGD and $\mathrm{LD}$, and ptosis occurs in LD.

Bell et $a^{6}$ identified FOXC2 mutations in 14 families with LD syndrome, all but 1 of them being small insertions or deletions, and Brice et $\mathrm{al}^{1}$ enlarged this number of families to 18 , plus 6 isolated cases. Erickson et $\mathrm{al}^{7}$ have also reported mutations in 7 families with variable expression of LD syndrome. Finegold et $a l^{9}$ found mutations in 10 families with LD and one family with lymphoedema without distichiasis. While the majority of reported FOXC2 mutations are small insertions/deletions, a nonsense mutation was identified in a large family with LD by Traboulsi et al. ${ }^{10}$ Three missense mutations have also been shown to produce LD. ${ }^{6,11}$ Bahuau et al ${ }^{12}$ identified a FOXC2 truncating mutation in a family with $\mathrm{LD}$ and cleft palate. A FOXC2 truncating mutation has been identified in affected individuals of a family with hereditary distichiasis but without lymphoedema or other features of LD syndrome. ${ }^{13}$

Since mutations in FOXC2 have been identified in families with lymphoedema-distichiasis syndrome, and it has been proposed that FOXC2 mutations may occur in lymphoedema with ptosis, lymphoedema with yellow nails, and Meige disease, ${ }^{9}$ we screened 23 patients $(17 \mathrm{~F}$ and $6 \mathrm{M})$ with pubertal onset lymphoedema and a suggested diagnosis of Meige disease, for mutations in FOXC2.

Our previous study on patients with $\mathrm{LD}^{1}$ showed that distichiasis is $94 \%$ penetrant. Lymphoedema is also highly penetrant, but it is difficult to give a figure for this as the age of onset is so variable and there were few individuals with FOXC2 mutations over 40 years in our cohort. It would intuitively appear that affected individuals would be aware of extra eyelashes, but this is not always the case. We have found several instances where individuals reported that they did not have distichiasis, including one where there was a full set of additional lashes. Three members of the family described in the present publication were unaware of their distichiasis. Rigorous phenotyping is thus essential in this condition.

\section{Subjects and methods}

One affected subject from 23 families was used to screen the FOXC2 gene. All families were ascertained based on the presence of primary lymphoedema in at least two family members. These consecutive patients attended a joint dermatology/genetics clinic, and were referred from clinicians throughout the United Kingdom. All were examined for the presence of distichiasis. Of these patients, 21 had between 1 and 7 living affected relatives, a total of 62, of which 12 were male patients.

\section{Lymphoscintigraphy}

This currently represents the gold standard screening test for lymphoedema. This was conducted in nine cases (Table 1). Imaging can help determine potency of lymph conducting pathways and distinguish apparent hypoplasia of peripheral lymphatics, for example, Milroy and Meige disease, from hyperplasia with reflux, for example LD, so defining the phenotypic abnormality further.

\section{Mutation detection}

Genomic DNA was extracted from peripheral venous blood by a standard procedure using a Nucleon genomic DNA extraction kit (Nucleon Biosystems). Mutation analysis of the single coding exon of the FOXC2 gene was carried out by PCR amplification and direct sequencing of three overlapping fragments. Primer sequences are available upon request.

\section{Results}

Sequencing of FOXC2 did not identify any mutation in 22 of 23 affected subjects. However, in one family (family 71 in Table 1) with 8 affected individuals in 3 generations, a 22-bp deletion was identified. This deletion includes nucleotides at position 563-584 (initiation ATG is numbered base 1 in the sequence with GenBank Accession no. Y08223). The forkhead domain of FOXC2 is reported to extend from nucleotides 211-510. The mutation described above is located only 17 amino acids after the forkhead domain and will lead to truncation of the mature protein. This truncation eliminates those regions located at the $\mathrm{C}$ terminus of the FOXC2 forkhead domain, which are necessary for the interaction of FOXC2 with the transcriptional complex. ${ }^{14}$ The deletion was verified by subcloning of PCR products from both mutant and wild-type alleles into a TA cloning vector (Invitrogen) followed by sequencing with M13 primers. The 22-bp deletion shifts the open reading frame and would create five novel amino acids before terminating with a premature stop codon at 
Table 1 Clinical details of the 23 cases and their family members

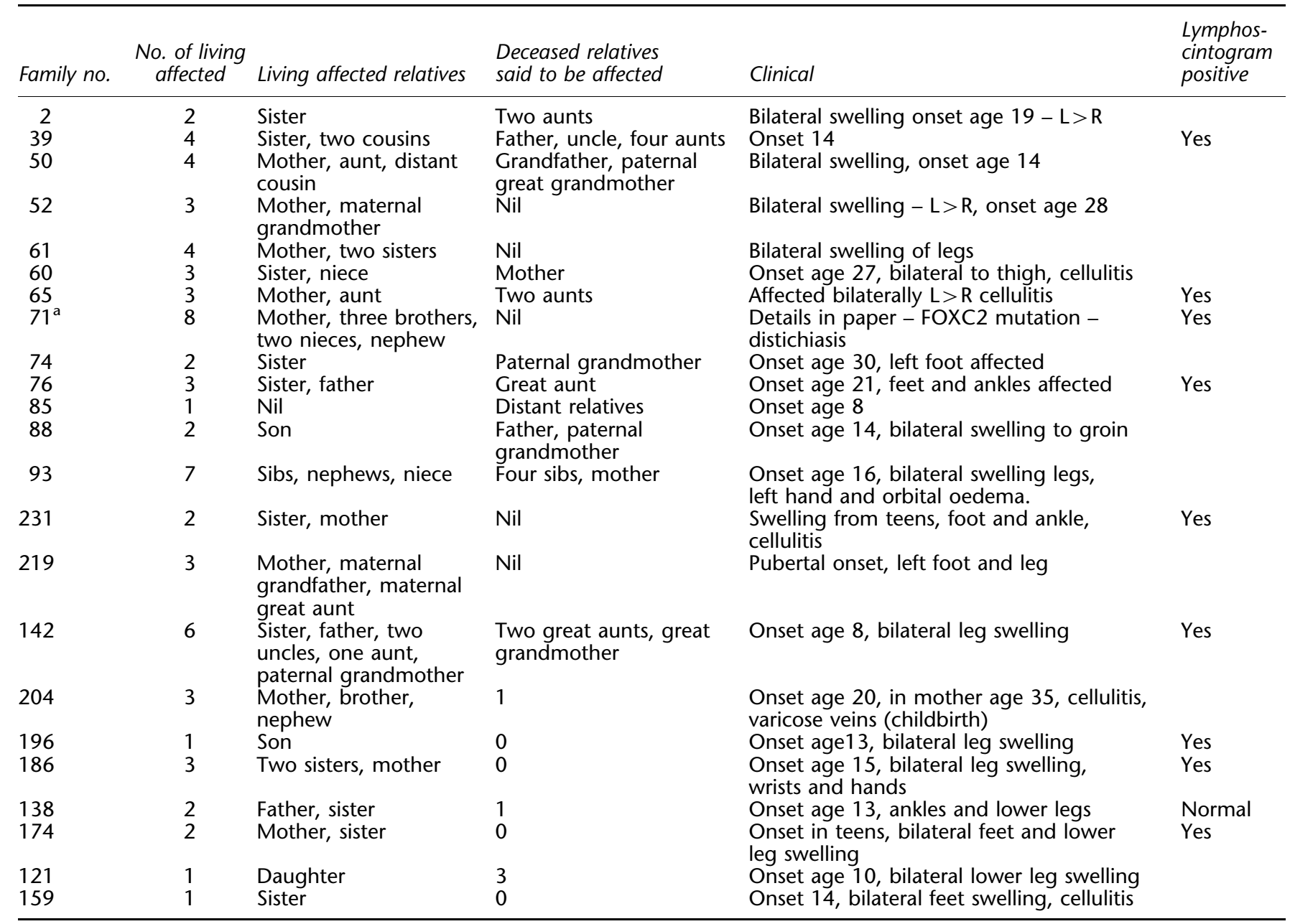

${ }^{a}$ Other than family 71 - no history of ptosis, distichiasis, congenital heart disease, cleft lip or palate as found in families with FOXC2 mutations.

nucleotide 599 or amino acid 200, truncating the normal protein by $38 \%$ (Figure 1), assuming a protein is produced from the mutated transcript. All affected members studied had the mutation, producing a 574-bp fragment, while none of the unaffected family members had the mutation and produced only a 596-bp fragment on PCR (Figure 1). Absence of this mutation in normal population was examined by screening of genomic DNA from 101 unrelated normal control subjects.

In this one family with the FOXC2 mutation, affected individuals had lymphoedema of varying severity with age of onset reportedly ranging from 12 to 35 years. Six individuals reported varicose veins (two females and four males) with onset between 14 and 20 years (one had a history of recurrent venous ulceration of the ankles), and three have undergone operative procedures for this problem. Four of the seven patients had lymphoscintigraphy; two proved to be positive bilaterally (II-2, II-5), one positive on the left only (II-4), with the fourth showing a rapid uptake of tracer (III-2), noted to be consistent with venous involvement. Six individuals have been examined carefully (I-2, II-2, II-4, II-5, III-1 and III-2) by an author (GB) for signs of distichiasis. Information for the other individual (III-5) has been gathered from medical notes and from the patient himself. Of the six individuals examined, four were found to have distichiasis. Individual II-5, who did not appear to have distichiasis on examination at home was seen by an ophthalmologist (SAV) and underwent slit lamp examination. No aberrant lashes were noted. Individual II-2 also had no evidence of distichiasis, but was not examined by an ophthalmologist.

In addition to the findings of lymphoedema and distichiasis, 1 patient (III-2), born at 31 weeks gestation, had a patent ductus arteriosus and pyloric stenosis and her father had a deep vein thrombosis. There was no evidence of other signs associated with FOXC2 mutations, such as 

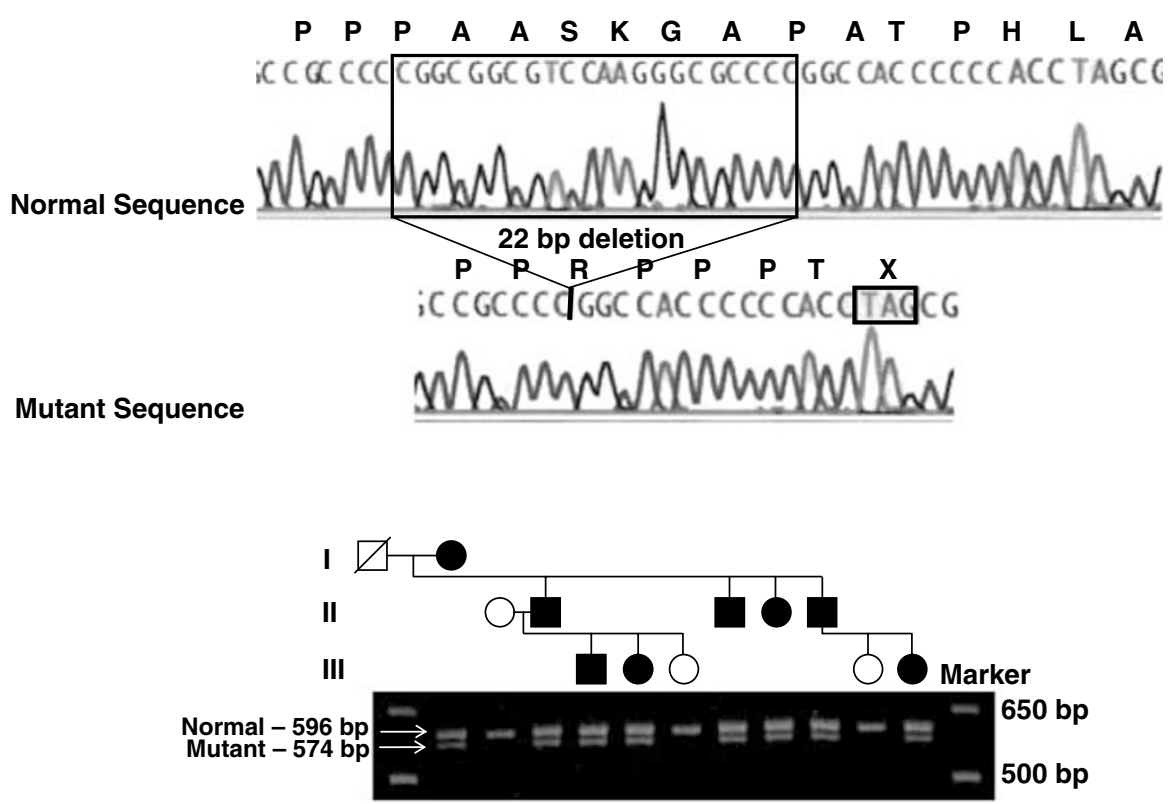

Figure 1 Sequence analysis and segregation of FOXC2 mutation; top: sequencing results from patient III-2 after cloning show the normal and mutant alleles, position of the 22-bp deletion and the predicted premature stop codon. Bottom: presence of mutant allele (574 bp) in all affected but not normal subjects.

cleft palate or ptosis, in these individuals or in any preceding generation.

\section{Discussion}

We studied the occurrence of mutations in the FOXC2 gene in 23 probands with non-congenital lymphoedema. The only mutation observed was a 22-bp deletion in a family with lymphoedema-distichiasis syndrome, while no FOXC2 mutation was identified in families with lymphoedema but without distichiasis.

It is important to know whether clinical differentiation of families with primary lymphoedema is reflected at the genetic level, as this has implications for which genes should be screened in the different phenotypic forms. In this respect, it is essential to distinguish between families in which a few members show symptoms that are major manifestations of other recorded syndromes, and families with the syndrome itself. For example, ptosis occurs in about $30 \%$ of LD patients. It is therefore not surprising that seven individuals in the cohort described by Finegold et al ${ }^{9}$ had ptosis, only two of whom reportedly had no distichiasis. Our experience would suggest that these are simply families with LD, with its known complex phenotype and variable expression, not examples of lymphoedema with ptosis (OMIM 153000). It may well be that no such entity as lymphoedema with ptosis actually exists, but until families where lymphoedema and ptosis are the predominant phenotypic features are examined, this remains an open question. Of the two references in OMIM which support the existence of the syndrome, that of Falls and Kertesz ${ }^{15}$ clearly describes a family with LD where distichiasis occurs in 10 of 11 affected individuals and is the predominant symptom, while ptosis is present only in two individuals. Indeed, FOXC2 mutations have already been identified in this pedigree. ${ }^{7}$ In the report of Bloom, ${ }^{16}$ there is no reference to the family members being examined for distichiasis, but the proband, aged 39, had varicose veins on feet and legs. It appears quite possible that this family had LD, with unusually high incidence of ptosis. If so, there would be no evidence of lymphoedema with ptosis as a separate syndrome.

Yellow discoloration of nails is not uncommon with lymphoedema but does not necessarily indicate YNS, where the nail changes are very specific. In YNS the nail plate is yellow and over-curved but it remains translucent and smooth whereas in lymphoedema not associated with YNS, the nails become thickened, rough and opaque. Associated features such as chronic sinusitis, bronchiectasis or pleural effusion are essential for a diagnosis of YNS. In four families said to have this syndrome Finegold et $a l^{9}$ found no mutation in FOXC2, although no clinical details of the families were given. In a series of 12 patients with YNS there was no family history of any phenotypic features, suggesting it may not even be genetic in origin. ${ }^{17}$

Both familial lymphoedema with ptosis, and YNS, if they exist, are very rare, but the possibility that mutations in FOXC2 occur in MGD is a much more important issue. In the families in our cohort that had been ascertained as MGD, we did find one where there was a mutation in FOXC2, but examination of additional family members 
showed that distichiasis was present in four out of the other six individuals with the mutation (individual II- 4 was not examined). Three of these individuals were unaware of the existence of the lashes. This was therefore a family with LD with two individuals who did not exhibit secondary lashes. A careful phenotypic study of our LD patients has shown that distichiasis is $94 \%$ penetrant, similar to the penetrance level of lymphoedema. ${ }^{1}$ In the series of patients presented by Finegold et $a l,{ }^{9}$ of the 48 who had lymphoedema (only 24 of whom were tested for the mutation), only 26 had distichiasis. The most likely explanation for this apparent low penetrance of distichiasis is the difficulty of getting reliable ophthalmic examination of patients in a study across the United States. It is mentioned by Finegold et $a l^{9}$ that medical records were requested to confirm medical diagnoses, suggesting that distichiasis could have been missed if only partial or not affecting the patient. This could well explain the family in that study ${ }^{9}$ where distichiasis is reportedly absent in all three affected members, which would make this a family with LD rather than Meige disease. Although we have never seen it in any of our families, it is possible that the three individuals in the study of Finegold et $a l^{9}$ did not have distichiasis. If so, they should still be classified as having LD, not Meige disease, because they have a FOXC2 mutation. We still believe it far more likely that ophthalmological examination of the three affected individuals in this family would show some degree of distichiasis in some of them.

In conclusion, we find that FOXC2 mutations are found only as part of the LD spectrum, but some symptoms of other proposed syndromes occur in some individuals with $\mathrm{LD}$, as the condition has a complex phenotype. In our LD families with FOXC2 mutations, the great majority of gene carriers have distichiasis, and we do not have any such families where distichiasis is absent. Sometimes, the distichiasis is not easy to identify even by experienced observers, let alone the patient, and careful phenotyping is essential in determining the clinical spectrum of the primary lymphoedemas. Asymptomatic distichiasis appears to be more common than might be expected, and relying on patient testimony or family hearsay as to the presence or absence of distichiasis should be avoided. After careful examination, advice on which genes to screen can be given. At present this would be VEGFR3 in MD, FOXC2 in $\mathrm{LD}$, however, no genes have yet been identified for MGD and YNS.

\section{Acknowledgements}

This study was supported by a grant from the National Institutes of Health (National Heart, Lung and Blood Institute; HL066150) to MS.
$R B$ was supported by the British Heart Foundation. The research was carried out within the network of the London IDEAS Genetic Knowledge Park, and used the resources of the Biomics Unit at SGUL. We thank the Reece Spence Fund and the London Law Trust for supporting this work. AC and GB thank St George's University of London and St George's Hospital NHS Trust for their support.

\section{References}

1 Brice G, Mansour S, Bell R et al: Analysis of the phenotypic abnormalities in lymphoedema-distichiasis syndrome in 74 patients with FOXC2 mutations or linkage to $16 \mathrm{q} 24$. J Med Genet 2002; 39: 478-483.

2 Mellor R, Brice G, Stanton A et al: Mutations in FOXC2 are strongly associated with primary valve failure in veins of the lower limb. Circulation 2007; 115: 1912-1920.

3 Evans AL, Brice G, Sotirova V et al: Mapping of primary congenital lymphedema to the 5q35.3 region. Am J Hum Genet 1999; 64: $547-555$.

4 Ferrell RE, Levinson KL, Esman JH et al: Hereditary lymphedema: evidence for linkage and genetic heterogeneity. Hum Mol Genet 1998; 7: 2073-2078.

5 Mangion J, Rahman N, Mansour S et al: A gene for lymphedemadistichiasis maps to 16q24.3. Am J Hum Genet 1999; 65: 427-432.

6 Bell R, Brice G, Child AH et al: Analysis of lymphoedemadistichiasis families for FOXC2 mutations reveals small insertions and deletions throughout the gene. Hum Genet 2001; 108: 546-551.

7 Erickson RP, Dagenais SL, Caulder MS et al: Clinical heterogeneity in lymphoedema-distichiasis with FOXC2 truncating mutations. J Med Genet 2001; 38: 761-766.

8 Fang J, Dagenais SL, Erickson RP et al: Mutations in FOXC2 (MFH1), a forkhead family transcription factor, are responsible for the hereditary lymphedema-distichiasis syndrome. Am J Hum Genet 2000; 67: 1382-1388.

9 Finegold DN, Kimak MA, Lawrence EC et al: Truncating mutations in FOXC2 cause multiple lymphedema syndromes. Hum Mol Genet 2001; 10: 1185-1189.

10 Traboulsi EI, Al-Khayer K, Matsumoto M et al: Lymphedemadistichiasis syndrome and FOXC2 gene mutation. Am J Ophthalmol 2002; 134: 592-596.

11 Sholto-Douglas-Vernon C, Bell R, Brice G et al: Lymphoedemadistichiasis and FOXC2: unreported mutations, de novo mutation estimate, families without coding mutations. Hum Genet 2005; 117: $238-242$.

12 Bahuau M, Houdayer C, Tredano M, Soupre V, Couderc R, Vazquez MP: FOXC2 truncating mutation in distichiasis, lymphedema, and cleft palate. Clin Genet 2002; 62: 470-473.

13 Brooks BP, Dagenais SL, Nelson CC et al: Mutation of the FOXC2 gene in familial distichiasis. J Aapos 2003; 7: 354-357.

14 Kaufmann E, Knochel W: Five years on the wings of fork head. Mech Dev 1996; 57: 3-20.

15 Falls HF, Kertesz ED: A new syndrome combining Pterygium colli with developmental anomalies of the eyelids and lymphatics of the lower extremities. Trans Am Ophthalmol Soc 1964; 62: 248-275.

16 Bloom D: Hereditary lymphedema (Nonne-Milroy-Meige). Report of a family with hereditary lymphedema associated with ptosis of the eyelids in several generations. N Y J Med 1941; 41: 856-863.

17 Hoque SR, Mansour S, Mortimer PS: Yellow nail syndrome: not a genetic disorder?. Eleven new cases and a review of the literature. Br J Dermatol 2007; 156: 1230-1234. 